Int. J. Curr. Res. Med. Sci. (2018). 4(7): 1-7

\begin{tabular}{|c|c|c|}
\hline International Journal of Current Research in & \\
Medical Sciences & ISSN: 2454-5716 \\
P-ISJN: A4372-3064, E -ISJN: A4372-3061 \\
WWW.ijcrims.com
\end{tabular}

\title{
Study of histomorphological patterns of ductal carcinoma in situ (DCIS) occurring simultaneously with infiltrating duct carcinoma (IDC) of breast in menopausal females
}

\author{
*Vikram Jassal, **Sarabjit Kaur, ***Vijay Kumar Bodal, \\ *****Manjit Kaur Mohi, ******N.S. Neki \\ * Senior Resident, Dept. of Pathology, Govt. Medical College, Patiala, India. \\ **Assistant Professor, Dept. of Obstetrics\& Gynaecology, GMC, Patiala, India. \\ ***Associate Professor, Dept. of Pathology, GMC, Patiala, India. \\ ****Professor \&Head, Dept. of Obstetrics \& Gynaecology, GMC, Patiala, India. \\ *****Professor of Medicine, Govt. Medical College, Amritsar, India \\ Corresponding author: Dr. Sarabjit Kaur, \\ Assistant Professor (Obstetrics and Gynecology), Govt. Medical College, Patiala, Punjab, India. \\ Email: sarbhjit_sandhu@yahoo.com
}

\begin{abstract}
Breast cancer ranks second according to the incidence of cancers among women in the world. The peak incidence of breast cancer in developed countries is above the age of 50 years whereas in India it is above the age of 40 . Ductal carcinoma in situ lies along a spectrum of preinvasive lesions originating within normal breast tissue, with histological progression from atypical hyperplasia to invasive breast cancer. DCIS is generally categorized by architectural description into five groups, comedo, solid, cribriform, papillary and micropapillary. Studies have found a significant association between nuclear grade of DCIS and histological grade of infiltrating carcinoma. In the present study, out of 60 cases, $33(55 \%)$ show a single growth pattern while $27(45 \%)$ showed a mixed growth pattern. In the present study, comedo pattern is most commonly observed in DCIS lesions with a single growth pattern $(48 \%)$, followed by solid pattern $(42 \%)$, and cribriform pattern $(06 \%)$. The micropapillary pattern was the least common pattern $(04 \%)$. In the present study, a highly significant correlation was found between the nuclear grade of DCIS and Bloom Richardson grade of infiltrating ductal carcinoma. The present study has validated the concept that nuclear grade of DCIS, as well as architectural pattern, are significantly correlated with the histological grade of invasive carcinoma.
\end{abstract}

Keywords: Breast, Carcinoma, Ductal, Menopause, Neoplasm 


\section{Introduction}

Cancer is one of the major health issues. Breast cancer is the most common cancer diagnosed in women throughout the world and is the most common cause of death in women. It ranks second according to the incidence of cancers among women in the world.[1] Breast cancer burden is increasing both in developing and developed countries; the peak incidence of breast cancer in developed countries is above the age of 50 years whereas in India it is above the age of 40.[2]

There has been a steady rise in the incidence of breast cancer worldwide. According to GLOBOCON 2012, in the previous five years, 1.7 million women were diagnosed with breast cancer and there were 6.3 million women alive who had been diagnosed with breast cancer. Since the 2008 estimates, breast cancer incidence has increased by more than $20 \%$, while mortality has increased by $14 \% .[3]$

Ductal carcinoma in situ (DCIS) is a proliferation of malignant-appearing cells of ducts and terminal lobular units of the breast that have not yet breached the basement membrane. DCIS was first recognized in the year 1930 but the concept that it could evolve into a malignant lesion was recognized in the year 1940.[4]Ductal carcinoma in situ lies along a spectrum of preinvasive lesions originating within normal breast tissue, with histological progression from atypical hyperplasia to invasive breast cancer.[5]

Although the initiating steps and precise pathway of breast tumorigenesis remain poorly defined, it appears that nearly all invasive breast cancers arise from in situ carcinomas. The presence of shared chromosomal changes in both ductal carcinoma in situ and synchronous, adjacent invasive cancers demonstrates their clonal evolutionary relationship.[6]

In the last several decades, the incidence of DCIS has increased dramatically, due largely to screening mammography. DCIS now accounts for approximately $20 \%$ of breast cancers diagnosed by mammography.[7] According to Markov model that incorporates data from multiple mammography screening trials estimates the incidence of DCIS that will progress into invasive breast cancer if untreated is 100-270 per 100000.[8]

The presently recognized pathological prognostic factors for local recurrence of DCIS are nuclear grade, presence/absence of necrosis and architectural patterns, size/extent of DCIS and distance of excision margins.[9] DCIS is generally categorized by architectural description into five groups, comedo, solid, cribriform, papillary and micropapillary.[10] Significant heterogeneity is observed between architectural patterns and mixed patterns can be seen in a single case.[11] Studies have found a significant association between nuclear grade of DCIS and histological grade of infiltrating carcinoma.[12]

In developing countries like India, the rate of detection of DCIS remains low due to lack of screening programs and diagnostic facilities in the periphery. Most of the patients remain asymptomatic and usually present with an invasive form of breast cancer, so the majority of ductal carcinoma in situ occurs in association with invasive carcinomas.

The purpose of this study is to determine the various histomorphological patterns of DCIS occurring simultaneously with infiltrating ductal carcinoma of the breast in menopausal females.

\section{Materials and Methods}

The present study is a retrospective study conducted in the Department of Pathology, Government Medical College and Hospital, Patiala. It included 60 cases of mastectomies and lumpectomies specimens of menopausal females, which were received in the Department of Pathology over a time period of 2012-2015. Mastectomy and lumpectomy specimens that do not contain ductal carcinoma in situ (DCIS) and the patients with lobular carcinoma were not included. Already treated, patients on chemotherapy and patients with previously done lumpectomy with remnant tumour were also not included in the study. 
The mastectomy and lumpectomy specimens were fixed in $10 \%$ formalin. Multiple sections were taken from representative areas and after processing, paraffin blocks were prepared. Sections from paraffin blocks were stained with Hematoxylin and Eosin stain. All sections were examined under the microscope.

\section{Observation and Results}

In the present study $53(88 \%)$ had undergone modified radical mastectomy and 7 cases (12\%) had local excision. The present study included women who have achieved menopause. The occurrence of DCIS with IDC was relatively infrequent in age $>60$ years $(27 \%)$ cases. (Table- 1$)$

Table -1: Age wise distribution of occurrence of DCIS with IDC. $(\mathrm{N}=60)$

\begin{tabular}{|l|c|c|}
\hline \multicolumn{1}{|c|}{ Age } & Number of cases & Percentage of cases \\
\hline $40-50$ & 18 & $30 \%$ \\
\hline $50-60$ & 26 & $43 \%$ \\
\hline $60-70$ & 11 & $19 \%$ \\
\hline$>70$ & 05 & $08 \%$ \\
\hline Total & 60 & $100 \%$ \\
\hline
\end{tabular}

In the present study, out of a total of 60 cases, 33 cases $(55 \%)$ show a single growth pattern while
27 cases $(45 \%)$ showed a mixed growth pattern. (Table-2)

Table -2: Patterns of DCIS. $(\mathrm{N}=60)$

\begin{tabular}{|l|c|c|}
\hline \multicolumn{1}{|c|}{ DCIS pattern } & Number of cases & Percentage of cases \\
\hline Single & 33 & $55 \%$ \\
\hline Mixed & 27 & $45 \%$ \\
\hline
\end{tabular}

In the present study, of the 33 cases of single pattern DCIS, a solid pattern was seen in 14 cases (42\%) whereas comedo, cribriform and micropapillary pattern was seen in 16 cases
(48\%), 2 cases $(06 \%)$ and one case (04\%) respectively. The most common pattern was comedo, which was seen in $48 \%$ of cases. (Table-3)

Table-3: DCIS with a single growth pattern. $(\mathrm{N}=33)$

\begin{tabular}{|l|c|c|}
\hline \multicolumn{1}{|c|}{$\begin{array}{c}\text { DCIS with single growth } \\
\text { pattern }(\mathrm{N}=33)\end{array}$} & Numbers of cases & Percentage of cases \\
\hline DCIS with comedo pattern & 16 & $48 \%$ \\
\hline DCIS with solid pattern & 14 & $42 \%$ \\
\hline DCIS with cribriform pattern & 02 & $06 \%$ \\
\hline $\begin{array}{l}\text { DCIS with micropapillary } \\
\text { pattern }\end{array}$ & 01 & $04 \%$ \\
\hline
\end{tabular}

In the present study, of the 27 cases of mixed type DCIS, solid and comedo mixed pattern DCIS was present in 14 cases (52\%), solid and cribriform mixed pattern DCIS was identified in 11 cases
(42\%) and cribriform with micropapillary mixed pattern DCIS was seen in 02 cases (6\%). (Table-4) 
Int. J. Curr. Res. Med. Sci. (2018). 4(7): 1-7

Table-4: DCIS with mixed growth pattern $(\mathrm{N}=27)$

\begin{tabular}{|l|c|c|}
\hline \multicolumn{1}{|c|}{ DCIS with mixed growth pattern $(\mathrm{N}=27)$} & Number of cases & Percentage of cases \\
\hline Solid + comedo & 14 & $52 \%$ \\
\hline Solid + cribriform & 11 & $42 \%$ \\
\hline Cribriform+ micropapillary & 02 & $06 \%$ \\
\hline
\end{tabular}

In the present study, of the 36 cases of high nuclear grade DCIS, 04 cases (11\%) were associated with Bloom Richardson grade-1 IDC, 13 cases $(37 \%)$ were associated with grade -2 IDC and 19 cases (52\%) were associated with grade-3 IDC. Of the 18 cases of intermediate grade, 04 cases $(22 \%)$ were found with grade-1 IDC, 09 cases (50\%) with grade-2 IDC and 05 cases $(28 \%)$ with grade-3 IDC. Out of the 06 cases of low-gradeDCIS, 05 cases $(83 \%)$ were associated with grade-1 IDC and 01 case (17\%) with grade- 2 IDC. (Table-5)

Table-5: Association of DCIS grading with Bloom Richardson grading of IDC.

\begin{tabular}{|l|c|c|c|}
\hline \multicolumn{1}{|c|}{ Bloom Richardson grade } & $\begin{array}{c}\text { High grade DCIS } \\
(\mathrm{N}=36)\end{array}$ & $\begin{array}{c}\text { Intermediate grade DCIS } \\
(\mathrm{N}=18)\end{array}$ & $\begin{array}{c}\text { Low grade DCIS } \\
(\mathrm{N}=06)\end{array}$ \\
\hline Grade -1 & $04(11 \%)$ & $04(22 \%)$ & $05(83 \%)$ \\
\hline Grade -2 & $13(37 \%)$ & $09(50 \%)$ & $01(17 \%)$ \\
\hline Grade -3 & $19(52 \%)$ & $05(28 \%)$ & 00 \\
\hline Total & 36 & 18 & 06 \\
\hline
\end{tabular}
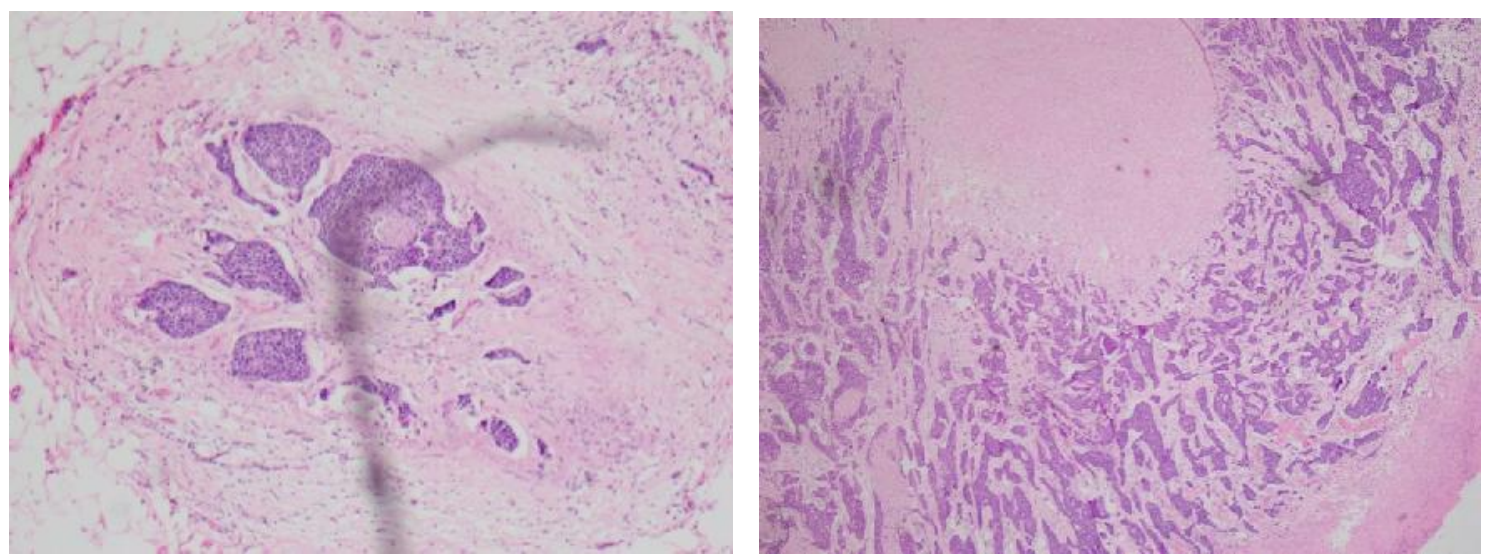

Fig 1: a) Solid pattern DCIS. b) Comedo pattern DCIS.
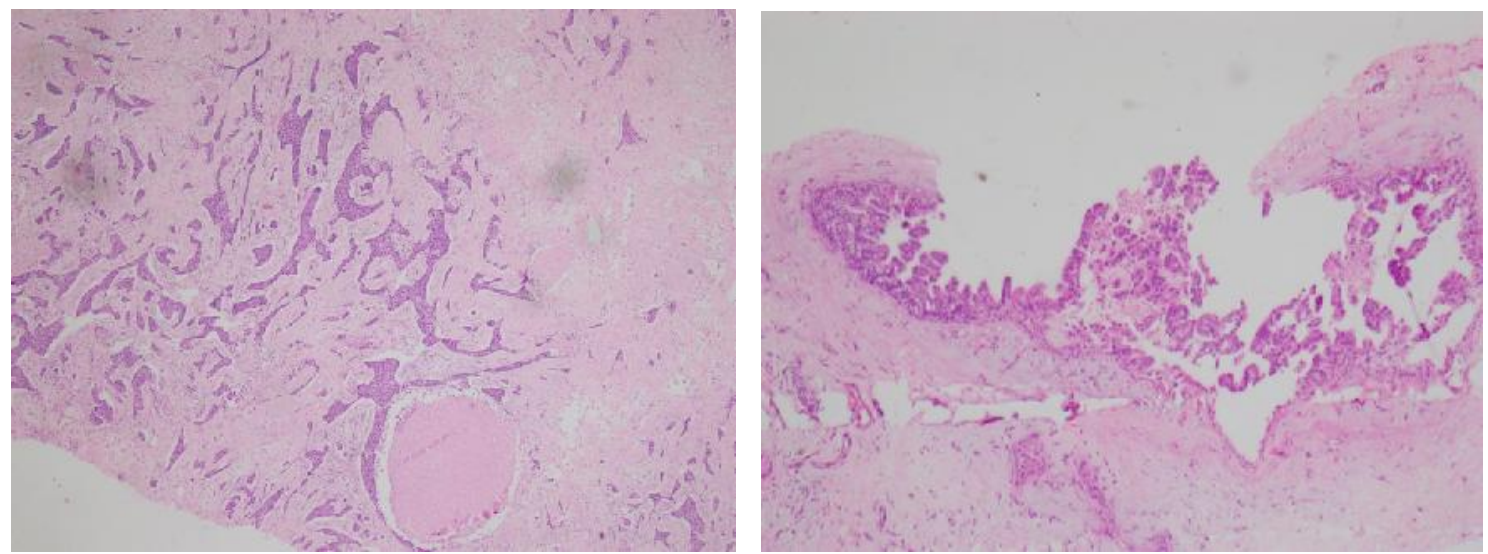

Fig 2: a) Mixed pattern DCIS comprising of solid pattern with comedo pattern.

b) Micropapillary pattern DCIS. 

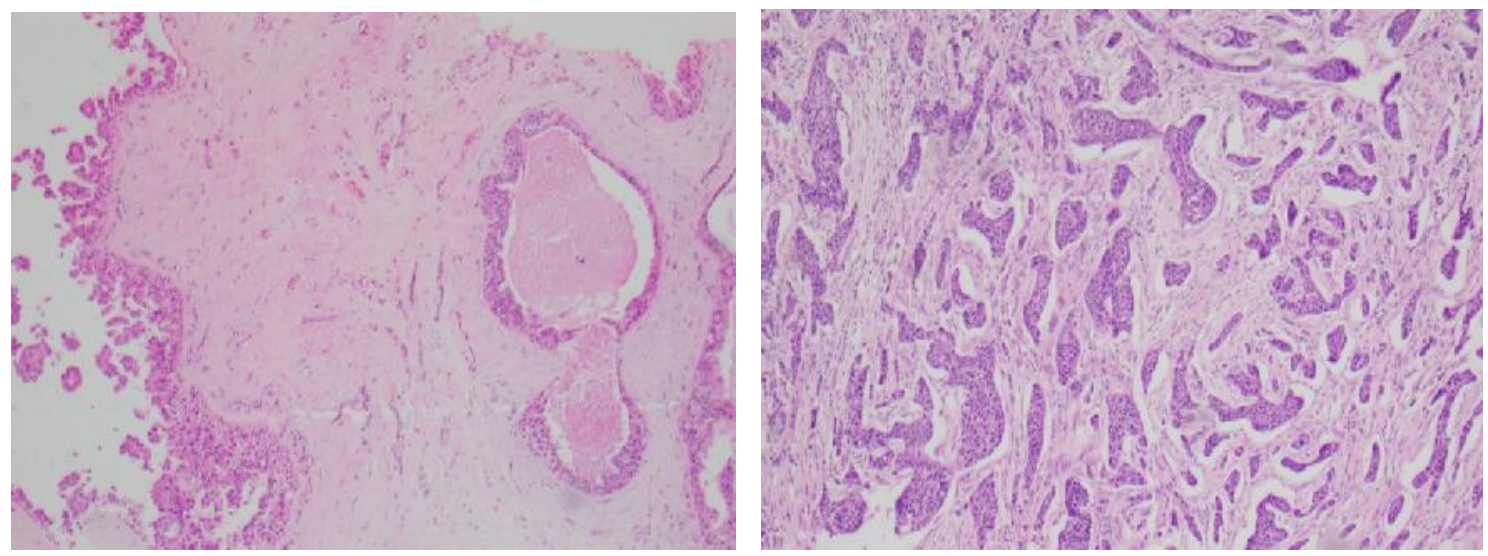

Fig 3: a) Mixed pattern DCIS comprising of micropapillary pattern with comedo pattern.

b) Solid pattern DCIS with the high nuclear grade.

\section{Discussion}

The present study was focused on various histomorphological patterns of DCIS and association between IDC of breast and nuclear grade of DCIS in menopausal females. DCIS was first noted in 1893, but the concept that it is a benign lesion, which could evolve into invasive cancer, was recognized in 1930.[13]. DCIS can occur as a pure lesion or can be found in association with invasive carcinoma regardless of stage and extension of the disease. Usually, DCIS is the precursor lesion of the infiltrating component.[14]

Various classification schemes have been used to grade DCIS, which are primarily based on architectural pattern, nuclear grade and the presence or absence of necrosis. These classification systems are useful in predicting the biological behaviour of DCIS as well in surgical management.[15] In the present study most of the patients are in the age range of 50 to 60 years and there is no significant difference in the age distributions of different structural subtypes of DCIS. However, Perez et al. showed that patients with high-grade DCIS were younger in age in relation to patients with low-grade DCIS. No such association is seen in the present study.[16]

In the present study, out of 60 cases, $33(55 \%)$ show a single growth pattern while $27(45 \%)$ showed a mixed growth pattern. This study is in agreement with the study by Scripcaru et al., which described the single growth pattern of
DCIS in 58\% cases and mixed growth pattern of DCIS in $42 \%$ of cases.[17]

In the present study, comedo pattern is most commonly observed in DCIS lesions with a single growth pattern $(48 \%)$, followed by solid pattern (42\%), and cribriform pattern (06\%). The micropapillary pattern was the least common pattern $(04 \%)$. These findings are in agreement with Lagios et al. who found that comedo type DCIS demonstrates the greater involvement of ducts as compared to cribriform and micropapillary.[18]Scripcaru et al. found $67 \%$ of cases of solid pattern DCIS, followed by $25 \%$ cribriform and $05 \%$ micropapillary cases.[17]

In the present study, a highly significant correlation was found between the nuclear grade of DCIS and Bloom Richardson grade of infiltrating ductal carcinoma. Grade -3 IDC (52\%) and grade-2 IDC (37\%) have a high nuclear grade of in situ component. In contrast, most of grade-1 IDC $(83 \%)$ are found to show low nuclear grade DCIS and none is found with high-grade DCIS. These findings are in strong agreement with Lampejo et al. who in their study have found a significant association between DCIS nuclear grade type and histological grade of infiltrating carcinoma. Low and intermediate grade DCIS are associated with Bloom Richardson grade -1 and grade -2 tumors respectively. High grade DCIS is associated with grade- 2 and grade- 3 infiltrating duct carcinoma.[18] 


\section{Conclusion}

The present study has evaluated heterogeneity of DCIS lesions associated with invasive carcinomas. Apart from single pattern DCIS, mixed pattern DCIS comprising of different architectural patterns can be seen within the same lesion. The present study has validated the concept that nuclear grade of DCIS, as well as architectural pattern, are significantly correlated with the histological grade of invasive carcinoma. Nuclear grade in combination with architectural pattern should be the best correlation with the grade of the invasive component.

\section{References}

1. Ferlay J, Shin HR, Bray F, Forman D, Mathers C, Parkin DM. GLOBOCAN 2008, Cancer incidence and Mortality Worldwide: IARC Cancer Base No. 10. Lyon, France: International Agency for Research on Cancer; 2010.

2. Yeole BB. Trends in cancer incidence in female breast, cervix uteri, corpus uteri and Ovary in india. Asian Pacific J Cancer Prev 2008;9:119-22.

3. Ferlay J, Soerjomataram I, Dikshit R, Eser S, Mathers C,Rebelo M, et al. Cancer incidence and mortality worldwide: sources, methods and major patterns in GLOBOCAN 2012.Int $\mathbf{J}$ Cancer. 2015;136(5):E359-86.

4. Olivotto I, Levine M, Steering Committee on Clinical Practice Guidelines for the Care and Treatment of Breast Cancer. Clinical practice guidelines for the care and treatment of breast cancer: the management of ductal carcinoma in situ (summary of the 2001 update). CMAJ. 2001;165(7):912-13.

5. Radford DM, Phillips NJ, Fair KL, Ritter JH, Holt M, Donis-Keller H. Allelic loss and the progression of breast cancer. Cancer Res 1995;55:5180-3.

6. Lakhani SR,Collins N, Sloane JP,Stratton MR. Loss of heterozygosity in ductal carcinoma in situ of the breast. Clin Mol Pathol 1995;48(2):M74-M78.
7. Ernster VL, Ballard-Barbash R, Barlow WE, Zheng Y, Weaver DL, Cutter G, etal. Detection of ductal carcinoma in situ in women undergoing screening mammography. J Natl Cancer Inst 2002;94(20):1546-54.

8. Kreger BE, Splansky GL, Shchatzkin A. The cancer experience in the Framingham Heart study cohort. Cancer 1991;67(1):1-6.

9. Pinder SE, Duggan C, Ellis IO, Cuzick J, Forbes JF, Bishop $\mathrm{H}$, et al. A new pathological system for grading DCIS with improved prediction of local Recurrence: results from the UKCCCR/ANZ DCIS tria. Br J Cancer 2010;103(1):94-100.

10. Rosai J. Borderline epithelial lesions of breast. Am J Surg Pathol 1991;15(3):209-21.

11. Consensus conference on the classification of ductal carcinoma in situ. The Consensus Conference Committee. Cancer 1997; 80(9):1798-1802.

12. Van Dongen JA, Holland R, Peterse JL, Fentiman IS, Lagios MD, Millis RR, et al. Ductal carcinoma in situ of the breast; second EORTC consensus meeting. Eur $\mathbf{J}$ Cancer 1992;28:626-9.

13. Lakhani SR, Ellis IO, Schnitt SJ, Tan PH, Van de Vijver MJ. World Health Organization Classification of Tumors of the Breast. $4^{\text {th }}$ edition. International Agency for Research on Cancer: Lyon; 2012. P. 240.

14. Siziopikou KP. Ductal carcinoma in situ of Breast: current concepts and future directions. Arch Pathol Lab Med 2013;137(4):462-6.

15. National Coordinating Group for Breast Screening Pathology. Guidelines for pathology reporting in breast cancer screening. NHSBSP Publication No. 3. Sheffield: NHS Breast Screening Programme, 1997:22-7.

16. Perez AA, Balabram D, Salles MA, Gobbi H. Ductal carcinoma in situ of the breast: correlation between histopathological features and age of patients. Diagn Pathol 2014;9:227.

17. Scripcaru G, Zardawi IM. Mammary ductal carcinoma in situ: a fresh look at architectural patterns. Int J Surg Oncol 2012; 2012:979521. 
18. Lagios MD, Westdahl PR, Margolin FR, Rose MR. Ductal carcinoma in situ. Relationship of extent of noninvasive disease to the frequency of occult invasion, multicentricity, lymph node metastases and short-term treatment failures. Cancer 1982; 50(7):1309-14.

\begin{tabular}{|c|l|}
\hline \multicolumn{2}{|c|}{ Access this Article in Online } \\
\hline Q & Website: \\
& www.ijcrims.com \\
\hline Quick Response Code & Subject: \\
\hline
\end{tabular}

How to cite this article:

Vikram Jassal, Sarabjit Kaur, Vijay Kumar Bodal, Manjit Kaur Mohi, N.S. Neki. (2018). Study of histomorphological patterns of ductal carcinoma in situ (DCIS) occurring simultaneously with infiltrating duct carcinoma (IDC) of breast in menopausal females. Int. J. Curr. Res. Med. Sci. 4(7): 1-7. DOI: http://dx.doi.org/10.22192/ijcrms.2018.04.07.001 